\title{
One-way edge mode in a magneto-optical honeycomb photonic crystal
}

\author{
Xianyu Ao, ${ }^{1, *}$ Zhifang Lin, ${ }^{2}$ and C. T. Chan ${ }^{1}$ \\ ${ }^{1}$ Department of Physics, Hong Kong University of Science and Technology, Clear Water Bay, Kowloon, Hong Kong, China \\ ${ }^{2}$ Surface Physics Laboratory and Department of Physics, Fudan University, Shanghai 200433, China \\ (Received 7 May 2009; revised manuscript received 25 June 2009; published 15 July 2009)
}

\begin{abstract}
We study the edge states in two-dimensional photonic crystals arising from a singular point in $k$ space at which two dispersion surfaces intersect. The zigzag edge states in a honeycomb lattice for TM polarization are analogous to the electronic ones in graphene nanoribbons. Electromagnetic modes at the zigzag edges of such photonic crystals consisting of ferrite rods are allowed to propagate only along one direction. The one-way propagation is insensitive to imperfections on the zigzag edge.
\end{abstract}

DOI: 10.1103/PhysRevB.80.033105

PACS number(s): 42.70.Qs

There has been strong interest in one-way propagating electromagnetic modes similar to the chiral edge states found in the quantum-Hall effect. ${ }^{1-4}$ These edge states are unique in that they allow for transport in only one direction and they are insensitive to scattering from imperfections. Realizing these states requires the breaking of time-reversal symmetry.

One-way electromagnetic modes can be realized in photonic crystals, which comprise a periodic arrangement of macroscopic media with different electromagnetic properties. ${ }^{5}$ Photonic crystals have provided us with various applications in the manipulation and control of light propagation. Up until now, a photonic crystal with a triangular lattice of gyroelectric materials for the TE polarization ${ }^{1}$ and a photonic crystal with a square lattice of gyromagnetic materials for TM polarization ${ }^{3}$ have been proposed to realize oneway electromagnetic wave propagation. The idea is to start with a band structure that has both time-reversal symmetry and inversion symmetry and which allows for the existence of "Dirac points" in the $k$ space. Dirac points are isolated points where two bands become degenerate but with a linear dispersion (resembling that of the massless Dirac equation) for nearby Bloch vectors. When a gap opens at the Dirac points due to time-reversal breaking, the two bands acquire nonzero Chern numbers resulting in one-way modes to occur at the edges of photonic crystals. ${ }^{1}$ However, the requirement for linear dispersion is not necessary for the realization of one-way edge modes. ${ }^{3}$

It is well known that Dirac points can be found in the electronic band structure of graphene which is a monolayer of graphite with carbon atoms packed into a two-dimensional (2D) honeycomb crystal structure. The electron transport in graphene is essentially governed by the Dirac equation and for a graphene ribbon (graphene with a finite width) with zigzag edges, there are peculiar localized electronic states at each edge. ${ }^{6}$ The corresponding energy bands are twofold degenerate and are nearly flat at the Fermi energy. The charge density in the edge state is strongly localized on the zigzag edge sites. On the other hand, such localized states do not exist in graphite systems having an armchair edge. We expect to see analogous features in electromagnetism..$^{7-9}$ In this Brief Report, we consider a zigzag ribbon of a magnetooptical photonic crystal (MOPC) of graphenelike (honeycomb) lattice (see Fig. 1) for TM polarization. We will show that there exist one-way edge states at each edge of the ribbon. These edge states support robust one-way transport for electromagnetic guided modes in the microwave regime. We note that our system is different from the case described in Ref. 3 where the MOPC was capped with another gapped material to form a waveguide. If the upper alumina photonic crystal is removed in the system described in Ref. 3, the propagating wave along the edge will disappear since their one-way modes lie inside the light cone. Here the one-way edge modes are exposed to free space. Even so, we will show that the one-way propagation is still insensitive to imperfections on the edge of the ribbon.

We start with the band structure of a two-dimensional honeycomb lattice of dielectric cylinders in air with TM polarization. For dielectric cylinders, the permittivity is $\epsilon_{m}$ $=15$ and the radius is $r=0.2 a$, where $a=10 \mathrm{~mm}$ is the lattice constant of the honeycomb lattice. The band structures are calculated by the multiple-scattering method and the details of the methodology are given in the literature. ${ }^{10,11} \mathrm{We}$ just remark here that the multiple-scattering method employs a linear combination of cylindrical harmonics to expand the fields inside and outside the cylinders and as the basis functions formally form a complete set (up to truncation errors), the results we obtain are highly precise. Figure 2(a) shows the band structure of our system. The first two dispersion surfaces intersect at a single point $\mathbf{K}$ in the Brillouin zone. This point is singular point in $k$ space. This quadratic degenerate point appears at $|k a|=2 \pi / 3$ when the band structure is projected onto a zigzag axis [see Fig. 2(b); the projected

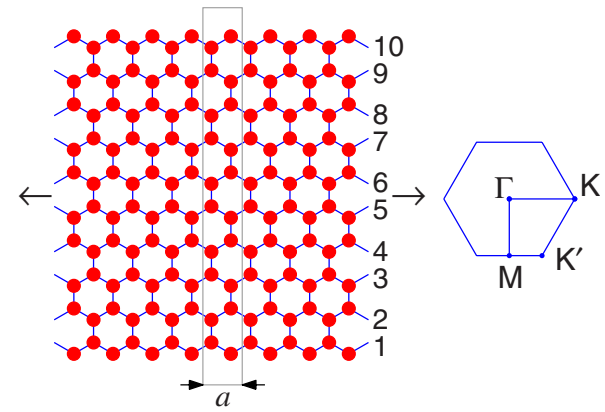

FIG. 1. (Color online) A zigzag ribbon with 10 zigzag chains. The arrows indicate the translational (periodic) directions of the ribbon and the rectangle indicates the unit cell of the ribbon. The right panel shows the first Brillouin zone. The band structure of the ribbon will be projected along the $\Gamma-\mathrm{K}$ direction. 


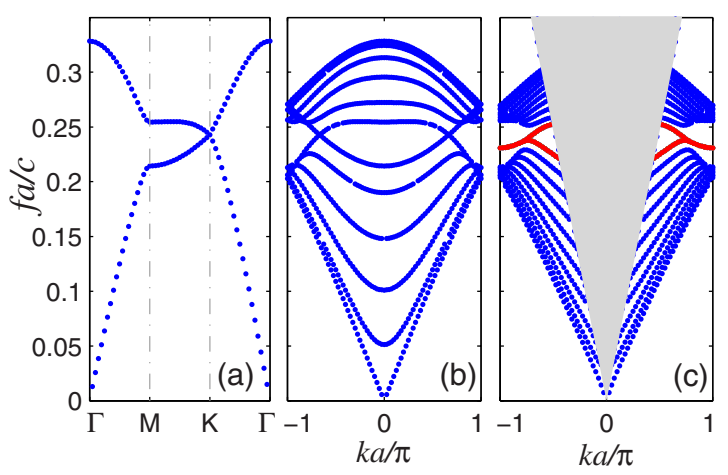

FIG. 2. (Color online) (a) Band structure for a 2D honeycomb lattice of dielectric rods with $\epsilon_{m}=15$ and $r=0.2 a$. (b) The corresponding projected band structure (of a supercell containing 10 primitive unit cells) onto a zigzag axis $(\Gamma-\mathrm{K})$. (c) The band structure of the ribbon shown in Fig. 1 along the $\Gamma-\mathrm{K}$ direction. The light cone is shaded and the edge states are shown in red (dark gray).

band structure is calculated with a supercell containing 10 copies of the primitive unit cell]. Figure 2(c) shows the band structure for the zigzag ribbon with 10 zigzag chains. The edge states, which appear inside a bulk gap, are plotted in red (dark gray). The two surface states are degenerate within the region of $2 \pi / 3 \leq|k a| \leq \pi$. This phenomenon is more obvious with increasing ribbon width. For a small ribbon width or a wave vector $k$, edge modes at two edges couple strongly, but they are more localized for larger values of the wave vector $k$ or bigger ribbon width. We note that the edge modes are outside the light cone and they form bonafide guided modes. They are completely localized at edge sites when $k a=\pi$ and start to gradually penetrate into inner sites as $|k a|$ deviates from $\pi$ reaching the extended states at $|k a|=2 \pi / 3$. This 2D photonic crystal can be viewed as an analog of graphene as far as edge states are concerned.

For comparison, we show in Fig. 3 the band structures similar to Fig. 2 but for a triangular lattice with TE polarization. There is also a singular point in $k$ space at which the second and third dispersion surfaces intersect. The dielectric constant and rod radius were chosen such that this singular point is below the light cone. We note that the two edge states arising from the singular point are degenerate within the region of $|k a| \leq 2 \pi / 3$ and extended into bulk states with

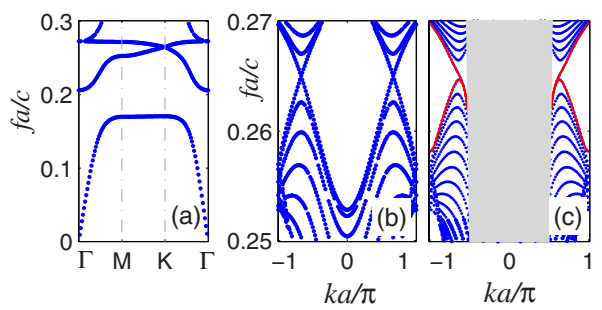

FIG. 3. (Color online) (a) Band structure for a $2 \mathrm{D}$ triangular lattice of dielectric rods with $\epsilon_{m}=40$ and $r=0.35 a$. (b) The corresponding projected band structure (of a supercell containing 20 primitive unit cells) onto the $\Gamma-\mathrm{K}$ direction. (c) The band structure along the $\Gamma-\mathrm{K}$ direction for a finite slab of 20 layers. The light cone is shaded and the edge states are shown in red (dark gray). increasing value of $|k a|$. This phenomenon is just the opposite to that of the honeycomb lattice for TM polarization. For the triangular lattice, a substantial part of the edge mode will go inside the light cone (shaded in Fig. 3) and will thus be leaky.

Breaking time-reversal symmetry lifts the degeneracy at $\mathbf{K}$ point and creates a gap. One convenient way to break the time-reversal symmetry is to employ gyromagnetic anisotropy, which can be implemented with commercially available ferrite rods for the TM polarization. When we apply a dc magnetic field along the axis of rods ( $z$ axis); the field induces the magnetic anisotropy of ferrimagnetic materials. When fully magnetized, the ferrite has the magnetic permeability tensor ${ }^{12}$

$$
\bar{\mu}=\left[\begin{array}{ccc}
\mu_{r} & -i \mu_{k} & 0 \\
i \mu_{k} & \mu_{r} & 0 \\
0 & 0 & 1
\end{array}\right],
$$

with

$$
\begin{gathered}
\mu_{r}=1+\frac{\omega_{m} \omega_{0}}{\omega_{0}^{2}-\omega^{2}}, \\
\mu_{k}=\frac{\omega_{m} \omega}{\omega_{0}^{2}-\omega^{2}},
\end{gathered}
$$

where $\omega_{0}=\gamma H_{0}$ is the precession frequency ( $\gamma$ is the gyromagnetic ratio and $H_{0}$ is the applied magnetic field) and $\omega_{m}=4 \pi \gamma M_{s}$ with $4 \pi M_{s}$ the saturation magnetization. We
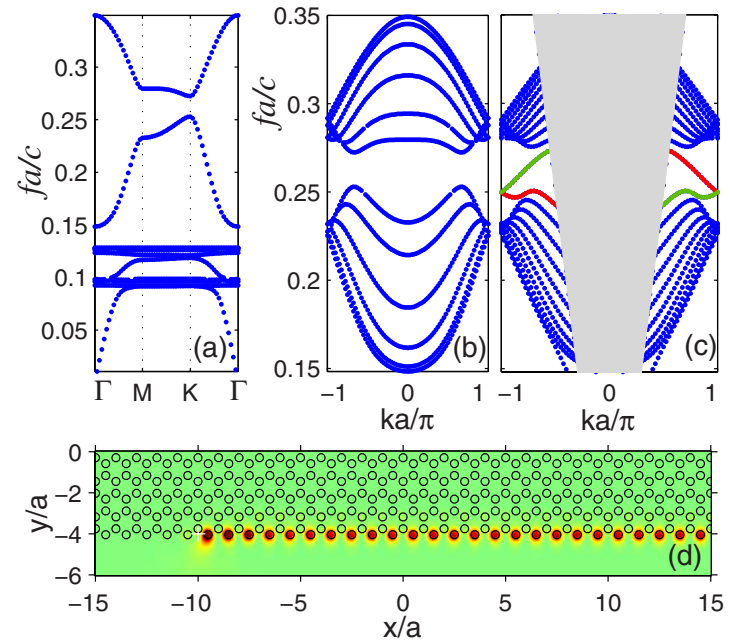

FIG. 4. (Color online) (a) Band structure for a honeycomb lattice of ferrite rods with $\epsilon_{m}=15, H_{0}=500 \mathrm{Oe}, 4 \pi M_{s}=1750 \mathrm{G}, r$ $=0.2 a$, and $a=10 \mathrm{~mm}$. (b) The corresponding projected band structure of a supercell containing 10 unit cells onto a zigzag axis $(\Gamma-\mathrm{K})$. (c) Band structure of the ribbon along the $\Gamma-\mathrm{K}$ direction. The light cone is shaded and the edge states are shown in red (dark gray) and green (light gray). (d) Squared amplitude of the $E_{z}$ field for one-way edge mode at one edge of the zigzag ribbon with 10 zigzag chains excited by a line source at $f a / c=0.254$ with green (light gray) and red (dark gray) representing zero and positive values. The line source is at $(-10,-4)$ (marked with "+" in the figure). The excited edge mode propagates to the right. 

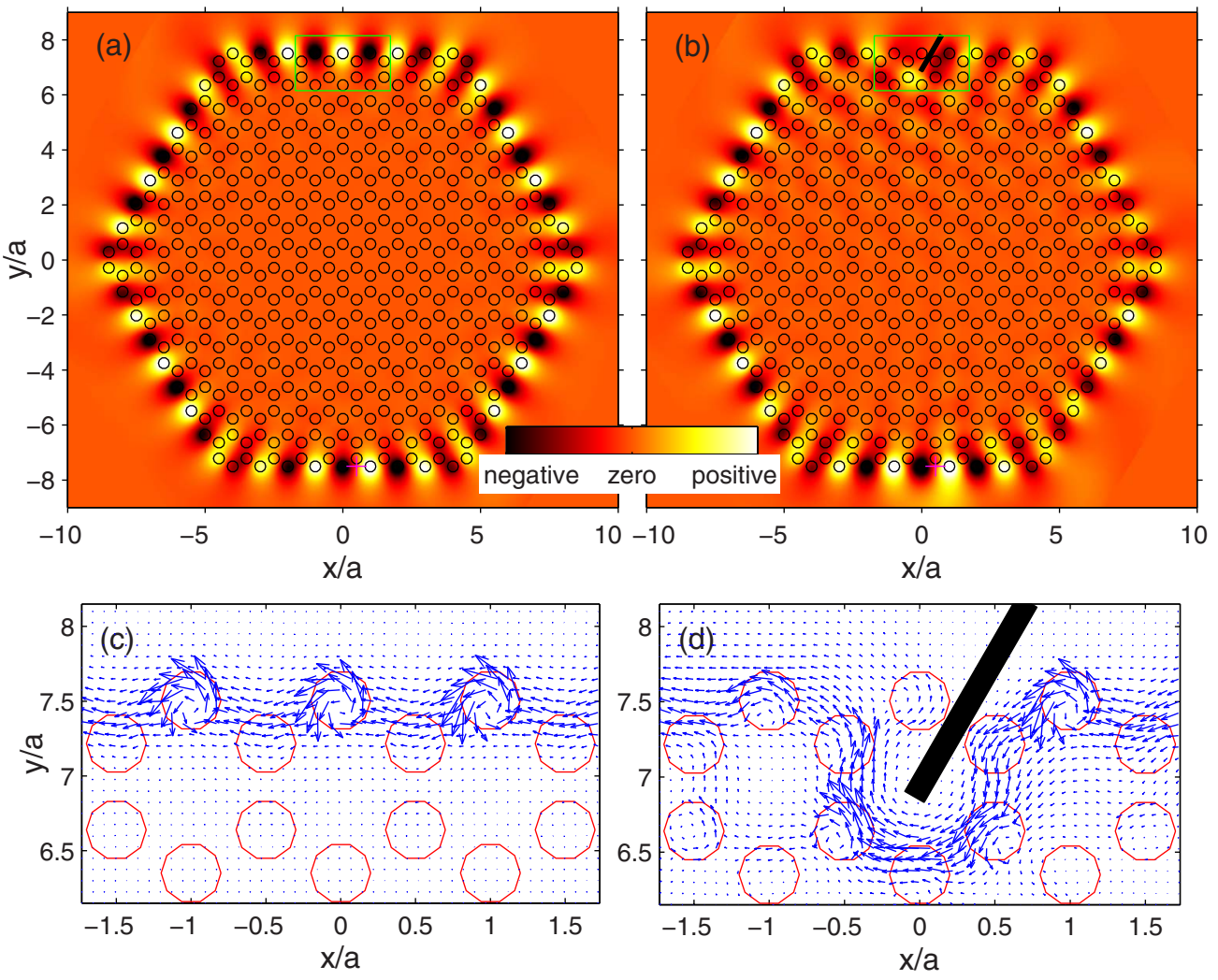

FIG. 5. (Color online) (a) $E_{z}$ field distribution for a hexagonal cavity consisting of ferrite rods excited by a line source at $f a / c$ $=0.254673$ (near the resonant frequency of the cavity) located at $(0.5,-7.5)$ (marked with " + " in the figure). There are nine rods at the outermost edge of the hexagon. The rods are the same as in Fig. 4. (b) Same as in (a) but with an obstacle inserted on the upper edge (black rectangle; a metal plate with thickness $0.15 a$ ). (c), (d) Distribution of Poynting vectors near the upper edge of the hexagon in (a) and (b), respectively. The power flow is cycling anticlockwise on the edges of the hexagon.

can define an effective permeability as $\mu_{e}=\left(\mu_{r}^{2}-\mu_{k}^{2}\right) / \mu_{r}$. It can be shown that in the ferrite material, the wave number is given by $k^{2}=\omega^{2} \mu_{e} \epsilon_{m}$. If we keep $\mu_{e}=1$ and increase $\mu_{k}$ gradually, we can see that the two edge bands in Fig. 2(c) will split with increasing strength of the time-reversal breaking $\left(\mu_{k} / \mu_{r}\right)$.

We show in Fig. 4 the results corresponding to an array of ferrite rods with $\epsilon_{m}=15, H_{0}=500 \mathrm{Oe}$, and $4 \pi M_{s}=1750 \mathrm{G}$. There parameters are typical for commercially available yttrium-iron-garnet. ${ }^{12,13}$ Figure 4(a) shows the band structure of the honeycomb lattice of ferrite rods. The dense flat bands around $f a / c=0.1$ are due to the resonance of $\mu_{r}$ and $\mu_{k}$. The frequency of interest is around $f a / c=0.25$ (for $a=10 \mathrm{~mm}$ the corresponding frequency is about $7.5 \mathrm{GHz}$ ) where we observe a small gap. The corresponding projected band structure (for a supercell containing 10 unit cells) along the zigzag axis is shown in Fig. 4(b). For the ribbon, edge states appear inside the gap of the infinite photonic crystal as shown in Fig. 4(c). The band structure of the ribbon is symmetric with respect to $k=0$ since the ribbon itself has inversion symmetry. If we cap the ferrite ribbon with another gapped material so that the two edges of the ribbon see different surroundings, we can see that the bands in green (light gray) are associated with one edge, while the bands in red (dark gray) are modes on the other edge. As a direct illustration of the one-way transport property, we see that waves from a line source near the edge of the ribbon only excite mode propagating to the right [see Fig. 4(d)]. The results are calculated by the finite-element frequency-domain method. The line source was located close enough to the edge to excite the edge states efficiently, since the edge states (as they are guided modes) can be excited by the evanescent wave components of the source.

As indicated in Fig. 4(d), the edge modes are strongly localized at edge sites. Such edge states can be used to construct an open cavity. Figure 5 shows a hexagonal cavity consisting of such ferrite rods in a honeycomb lattice bounded by zigzag edges. The mode shown in Fig. 5(a) is excited by a line source radiating at the frequency near one of the resonances of the cavity $(f a / c=0.254673)$. Figure 5(a) shows that the fields corresponding to the mode are strongly localized on the edge cylinders, as expected from the results shown in Fig. 4(d). Figure 5(c) shows the energy flow by showing the distribution of the Poynting vectors near the upper edge of the hexagonal array corresponding to the region outlined by the rectangle in Fig. 5(a). By examining the distribution of the Poynting vectors, we see that the power flow is cycling inside the ferrite rods on edge sites and such microscopic cycles form macroscopically the anticlockwise loop on the edges of the whole hexagon. We expect that the one-way power-flow characteristics should make the power flow robust against imperfections or disruptions along the edge. This is indeed the case as can be seen in Fig. 5(b). If we insert a metal plate (size $0.15 a \times 1.5 a)$ on the edge of the 
hexagon, as shown in Fig. 5(b), the power flow is still cycling on the edges of the hexagon. The microscopic details are shown in Fig. 5(d). The distribution of Poynting vectors in Fig. 5(d) shows that the power flow simply chose another route to go around the obstacle to avoid being scattered. For edge states supported by dielectric photonic crystals, such a drastic obstacle would introduce strong backscattering and completely destroy the guiding.

In conclusion, we demonstrated that one-way edge modes exist in a photonic crystal consisting of ferrite rods (under an external dc magnetic field) arranged in a honeycomb lattice. To achieve this we start with a lattice of dielectric rods which has a singular point in dispersion surfaces. These edge modes are below the light line so they are localized on the edges of a finite-size sample in free space. The edge modes can be excited by the evanescent fields of a line source. The oneway propagation is robust against obstacles on the edges of the photonic crystals.

This work is supported by Hong Kong RGC under Grant No. 600308. Z.F.L. acknowledges support from the China 973 Program, NSFC, MOE of China (Grant No. B06011), and PCSIRT. We thank Wei Ren, Dezhuan Han, Zhihong Hang, and Yun Lai for useful discussions.

\footnotetext{
*aoxy@ust.hk

${ }^{1}$ F. D. M. Haldane and S. Raghu, Phys. Rev. Lett. 100, 013904 (2008).

${ }^{2}$ S. Raghu and F. D. M. Haldane, Phys. Rev. A 78, 033834 (2008).

${ }^{3}$ Z. Wang, Y. D. Chong, J. D. Joannopoulos, and M. Soljacic, Phys. Rev. Lett. 100, 013905 (2008).

${ }^{4}$ Z. Yu, G. Veronis, Z. Wang, and S. Fan, Phys. Rev. Lett. 100, 023902 (2008).

${ }^{5}$ J. D. Joannopoulos, S. G. Johnsom, J. N. Winn, and R. D. Meade, Photonic Crystals, 2nd ed. (Princeton, New Jersey, 2008),

${ }^{6}$ K. Nakada, M. Fujita, G. Dresselhaus, and M. S. Dresselhaus,
}

Phys. Rev. B 54, 17954 (1996).

${ }^{7}$ O. Peleg, G. Bartal, B. Freedman, O. Manela, M. Segev, and D. N. Christodoulides, Phys. Rev. Lett. 98, 103901 (2007).

${ }^{8}$ H. Benisty, Phys. Rev. B 79, 155409 (2009).

${ }^{9}$ D. Han, Y. Lai, J. Zi, Z.-Q. Zhang, and C. T. Chan, Phys. Rev. Lett. 102, 123904 (2009).

${ }^{10}$ K. M. Leung and Y. Qiu, Phys. Rev. B 48, 7767 (1993).

${ }^{11}$ P. Chen, R. X. Wu, J. Xu, A. M. Jiang, and X. Y. Ji, J. Phys.: Condens. Matter 19, 106205 (2007).

${ }^{12}$ D. M. Pozar, Microwave Engineering, 2nd ed. (Wiley, New York, 1998), pp. 497-517.

${ }^{13}$ Handbook of Magnetic Materials, edited by E. Wohlfarth (North Holland, Amsterdam, 1980), Vol. 2, p. 293. 